\title{
Codo de tenista: en el mediano plazo la fisioterapia y los ejercicios de rehabilitación serían mejores a la infiltración con corticoides
}

Tennis elbow: physiotherapy and rehabilitation exercises appear to be better in the medium term than corticosteroid injection

Bisset L y col. BMJ 2006 Nov 4; 333(7575):939.

\section{Objetivo}

En el tratamiento del codo de tenista (CT) o "epicondilitis externa o lateral" comparar la eficacia a las 52 semanas de la fisioterapia (FT) la conducta expectante (CE) y la infiltración con corticoides (IC).

\section{Diseño, lugar y pacientes}

Estudio aleatorizado controlado y simple ciego, conducido en Brisbane, Australia. Participaron 198 pacientes de 18 a 65 años con diagnóstico clínico de CT de más de seis meses de duración a quienes no se les hubiera prescripto tratamientos durante los seis meses previos.

\section{Intervención}

Fueron aletorizados en tres grupos: $33,8 \%$ a CE; $32,8 \%$ a IC y $33,4 \%$ a FT. A los asignados a CE se los reaseguró, se les dio instrucciones específicas sobre como modificar sus actividades diarias y se les permitió usar calor, frío, analgésicos y coderas según lo necesitaran. Al los asignados a IC, se les inyectó $1 \mathrm{~mL}$ de lidocaína y $10 \mathrm{mg}$ de triamcinolona en los puntos dolorosos del codo, con una eventual segunda inyección a las dos semanas a criterio médico. Los asignados a FT realizaron ocho sesiones de 30 minutos durante seis semanas, se les enseñó ejercicios y movimientos para realizar en la casa, y recibieron equipamiento y un folleto con instrucciones para realizar los ejercicios.

\section{Medición de resultados principales}

Mejoramiento global, fuerza de la compresión de la mano o "grip" y evaluación del rango de severidad, con evaluación basal, a las seis y a las 52 semanas, y análisis por intención de tratar.

\section{Resultados principales}

Comparada con la FT, la IC mostró significativamente mejores resultados a las seis semanas pero con un alto índice de recu- rrencia posterior y significativamente peores resultados en el largo plazo.

En el corto plazo, la FT fue superior a la CE, sin diferencias a las 52 semanas. Ver tabla 1.

Tabla 1: resultados clínicos a las seis y a las 52 semanas de los pacientes asignados a conducta expectante (observación) infiltración con corticoides y fisioterapia.

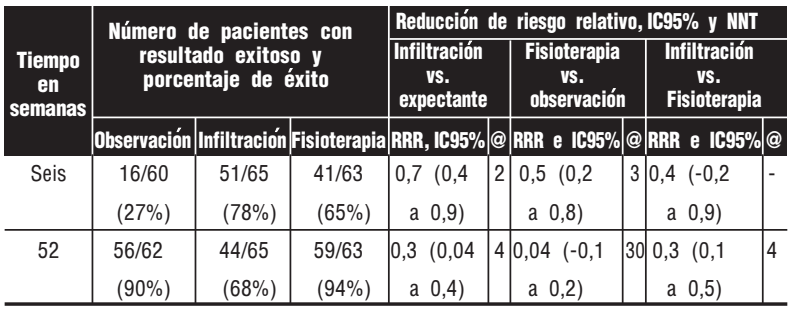

@NNT: número de pacientes necesario tratar.

Los participantes asignados a FT solicitaron menos tratamientos fuera del protocolo (ej. AINE) que los de los otros dos grupos.

\section{Conclusiones}

La FT con rehabilitación es más beneficiosa que la CE durante las primeras seis semanas y que la IC, luego de la sexta semana, generando una alternativa razonable a la IC en el mediano plazo. Paradójicamente, los pacientes sometidos a IC tienen alta probabilidad de recaída en el mediano plazo, lo que implica que este tratamiento debería ser utilizado con precaución en el CT.

Palabras clave: fisioterapia, infiltración con corticoides, conducta expectante, codo de tenista.

Key words: physiotherapy, steroid injection, expectant behavior, tennis elbow. Fuente de financiamiento: University of Queensland and the National Health and Medical Research Council, Primary Health Care Project Grant, Australia.

\section{Comentario}

EI CT afecta a entre 1 y $3 \%$ de la población general y a $15 \%$ de las personas que realizan una actividad de mayor riesgo'. Actualmente y con el objetivo de aliviar el dolor ${ }^{2}$, se propone el tratamiento con hielo o calor local, masajes con pomadas antiinflamatorias y una banda elástica de antebrazo con una placa rígida colocada sobre los músculos epicondíleos a $6 \mathrm{~cm}$ del epicóndilo; reservándose la IC cuando el dolor persiste luego de estas medidas.

Este estudio nos informa que la FT combinada con manipulación y ejercicio -siempre que pueda reproducirse la técnica evaluada en esta investigación- es mejor que a la CE en sus resultados precoces y que las IC en sus resultados a mediano plazo; y nos muestra también la importancia de la folletería con información sobre el manejo del CT y con consejos de autocuidado.
Si bien no se desprende directamente de los resultados de esta investigación, con el objetivo de lograr un rápido alivio inicial pareciera razonable desde el sentido común comenzar con una IC -que tiene los mejores resultados en el corto plazo- continuando luego con FT combinada con manipulación y ejercicios -que se asocia a mejores resultados en el mediano plazo.

\section{Conclusión de la comentadora}

Dado que la mayoría de los pacientes con CT mejoran en el mediano plazo, es difícil decidir la mejor estrategia terapéutica. Sería razonable explicarles los pros y los contras de cada alternativa para tengan las mejores herramientas para decidir.

Mercedes Mutchinick [ Servicio de Medicina Familiar del Hospital Italiano de Buenos Aires. mercedes.mutchinick@hospitalitaliano.org.ar ]

Mutchinick M. Codo de tenista: en el mediano plazo la fisioterapia y los ejercicios de rehabilitación serían mejores a la infiltración con corticoides. Evid. actual. práct. ambul; 11(4): 104, Jul-Ago.2008. Comentado de: Bisset L y col. Mobilisation with movement and exercise, corticosteroid injection, or wait and see for tennis elbow: randomised trial. BMJ 2006 Nov 4; 333(7575):939. PMID: 17012266.

Disponible en URL: http://www.bmj.com/cgi/reprint/333/7575/939

\section{Referencia}

1. Soriano E. Problemas comunes del codo, la muñeca y la mano. Capítulo 214. En: "Medicina Familiar y Practica Ambulatoria". Editores Rubinstein A. Terrasa S, Carrete P. Editorial Médica Panamericana, Marzo de 2001. pp1639.

2. Méndez Naya, B; Quiroga Dotras, R; Vázquez Millán, P; Louro González, A. Epicondilitis. Guías Clínicas 2007; 7 (16). Disponible en URL con registro gratuito desde Internet en: http://www.fisterra.com/guias2/epicondilitis.asp (último acceso 29-07-08). 